\title{
FIXED POINTS ARISING ONLY IN THE GROWTH OF FIRST COUNTABLE SPACES
}

\author{
STEPHEN WATSON
}

(Communicated by Franklin D. Tall)

\begin{abstract}
We construct a Tychonoff first countable space $X$ and an autohomeomorphism $f$ with no fixed points (either a translation or a reflection) such that $\beta f$ does have a fixed point answering a question of Krawczyk and Steprāns. We do this by replacing each point of Mrowka's construction of a first countable space whose growth has size one with a copy of the integers which can be translated.
\end{abstract}

\section{INTRODUCTION}

Fundamental to topology and particularly to its applications to differential equations, economics, and physics is the topic of fixed points of mappings. A basic result in the theory of Stone-Čech compactification is the fact that any autohomeomorphism $f$ of $X$, where $X$ is a Tychonoff space, can be extended uniquely to an autohomeomorphism $\beta f$ of $\beta X$.

If $f$ is an autohomeomorphism which has no fixed points, then it may still happen that $f$ has an "ideal" fixed point or that any extension of $f$ to an autohomeomorphism of a compactification has a fixed point. That is, it may happen that $\beta f$ does have a fixed point.

Definition 1. If $X$ is a Tychonoff space for which any autohomeomorphism $f$ with no fixed points has an extension $\beta f$ without fixed points, we say that $X$ is fixed-point-free autohomeomorphisms extends or FAE.

Blaszczyk, Kim, Krawczyk, and Steprāns have tried to determine which spaces are FAE spaces. It turns out that this can be quite difficult to determine even in some simple cases; however, they were able to obtain the following results.

Proposition 1 (Krawczyk and Steprāns [4]). Discrete spaces are FAE.

Proposition 2 (Blaszczyk and Kim [1]). Removing one point from $2^{\omega_{1}}$ leaves a space which is not FAE.

Received by the editors January 23, 1991 and, in revised form, February 1, i 793.

1991 Mathematics Subject Classification. Primary 54H25; Secondary 54C50, 54D35, 54C20, 54D30.

This work was supported by the Natural Sciences and Engineering Research Council of Canada. 
Since the gap between Propositions 2 and 1 is quite large, Krawczyk and Steprāns asked the following question.

Problem 1. Is there a first countable zero-dimensional space which is not FAE?

They were able to answer Problem 1 at least consistently by means of an Ostaszewski construction.

Theorem 1 (Krawczyk and Steprāns [4]). Under the assumption of Jensen's axiom $\diamond$, there is a first countable space which is not FAE.

In 1990 Steprāns resolved the case of the rationals.

Theorem 2 (Steprāns [4]). The rationals are FAE.

The case of the irrationals for self-maps rather than autohomeomorphisms was resolved by Mazur in 1990.

Theorem 3 (Mazur [5]). The irrationals have a (nononto) self-map $f$ which has no fixed point so that $\beta f$ has a fixed point.

In 1991 the files of the late Eric van Douwen yielded much stronger results.

Theorem 4 (van Douwen [2]). Any paracompact strongly zero-dimensional space is $F A E$.

Thus any zero-dimensional separable metric space is FAE, and, in particular, the case of the irrationals is solved positively.

Theorem 5 (van Douwen [2]). There is a locally compact separable metric space which is not FAE.

This example is just the disjoint union of the $n$-spheres. Of course, this example is not zero dimensional.

With all these results, Problem 1 remained open. We shall answer Problem 1 by constructing, in ZFC, two first countable spaces which are not FAE.

The fixed-point-free autohomeomorphisms $f$ whose Stone-Čech extension have a fixed point also have an algebraic structure. The orbit of each point is either finite or infinite. Indeed we can make some definitions to describe the two simplest cases.

Definition 2. If $f$ is an autohomeomorphism of a topological space $X$ and the orbits of each $x \in X$ have size 2, then we say $f$ is a reflection. Equivalently, $f$ is a reflection if $(\forall x \in X) f^{2}(x)=x \neq f(x)$.

Definition 3. If $f$ is an autohomeomorphism of a topological space $X$ and the orbits of each $x \in X$ have infinite size, then we say $f$ is a translation. Equivalently $f$ is a translation if $(\forall x \in X)(\forall n>0) f^{n}(x) \neq x$.

First, we shall construct a first countable space whose orbits are of size 2, that is, a reflection. Second, we shall construct a (much more subtle) first countable space whose orbits are infinite, that is, a translation.

The second construction is an adaptation of a beautiful argument of Mrowka on almost disjoint families to what we call translation almost disjoint families.

\section{A FIRST COUNTABLE EXAMPLE OF REFLECTION}

Theorem 6. There is, in ZFC, a first countable space which is not FAE; indeed, there is a reflection $f$ of $X$ such that $\beta f$ has a fixed point. 
Proof. In 1976 van Douwen [3] constructed a first countable Tychonoff space $X$ with two disjoint unseparated copies of $\omega_{1}, A_{0}$, and $A_{1}$. We shall first carry out some standard modifications of $X$. Declare $B=X-\left(A_{0} \cup A_{1}\right)$ to consist of isolated points. As Simon did, in his correction of Jones, take another copy of $X$ and identify $A_{i}$ from one copy with $A_{1-i}$ from the other copy. Now we have a new space $Y$ with underlying set $A_{0} \cup A_{1} \cup(B \times 2)$. The mapping $\pi$ which interchanges $A_{0}$ and $A_{1}$ and interchanges $B \times\{0\}$ and $B \times\{1\}$ is an autohomeomorphism of $Y$.

Now we argue that the closure of each $A_{i}$ in the Stone-Čech compactification adds only one point to $A_{i}$. It is clear that these points must be interchanged by $\beta f$. If these points are identical, then that is a fixed point and we are done. If these points $p_{i}$ are distinct, then a Hausdorff-witnessing separation of $p_{0}$ and $p_{1}$ traces down to a separation of a tail of $A_{0}$ and $A_{1}$. Since the rest of the $A_{i}$ 's is compact, we have a contradiction to the nonexistence of a separation of $A_{0}$ from $A_{1}$.

\section{A FIRST COUNTABLE EXAMPLE OF TRANSLATION}

Theorem 7. There is, in ZFC, a first countable space which is not FAE; indeed, there is a translation $f$ of $X$ such that $\beta f$ has a fixed point.

Definition 4. If $A \subset \omega$, we define $A+n$ to be $\{a+n: a \in A\}$ and call it a translate of $A$ where $n \in \mathbb{Z}$. If $\mathscr{G} \subset \mathscr{P}(\omega)$, we define $T(\mathscr{G})=\{A+n: n \in \mathbb{Z}$, $A \in \mathscr{G}$.

Definition 5. We say that two subsets $A$ and $B$ of $\mathbb{Z}$ are translation almost disjoint (t.a.d.) if $|(A+n) \cap B|=\omega$ implies $A=B$ and $n=0$.

Note that this definition is symmetric for $A$ and $B$.

Lemma 1. If $G$ is a maximal t.a.d. family, then $T(G)$ is a maximal almost disjoint family.

Lemma 2. Any $\psi$-space induced by $T(G)$ where $G$ is a maximal t.a.d. family has a fixed-point-free autohomeomorphism.

Proof. Map $z \in \mathbb{Z}$ to $z+1$, and send $A$ to $A+1$.

Lemma 3. If $X$ is a Tychonoff space, $f: X \leftrightarrow X$ is an autohomeomorphism, and $|\beta X-X|=1$, then $\beta f$ has a fixed point.

Proof. The range of a compact space must be compact, and we can only add one point to domain and range.

Lemma 4 (Mrowka, Remark 3.9). If $G$ is an infinite maximal almost disjoint family of subsets of $\omega, X$ is the $\psi$-space induced by $G$, and $E \subset G$ is an infinite zero set in $X$, then $E$ is uncountable.

Proof. Mrowka refers us to Gillman and Jerison's textbook exercise 51 on p. 79.

Lemma 5. If $G$ is an almost disjoint family of subsets of $\omega$, there is an injection $\phi: \omega \rightarrow \omega$ such that $\phi(G)$ is a t.a.d. family.

Proof. Define $\phi(n)=2^{n}$.

Lemma 6 (Variation on Mrowka). If $F$ is a family of continuum many functions on the $c \times \mathbb{Z}$, then there is a permutation $\pi$ of $c \times \mathbb{Z}$ such that if $f \in F$ and 
$f$ is not almost constant (modulo $<c$ ), then $f \circ \pi$ does not belong to $F$ and such that $\pi(c, z)+i=\pi(c, z+i)$.

Proof. Let $F_{0}$ be the subset of $F$ which consists of those functions which are not almost constant (modulo $<c$ ). For each $f \in F_{0}$, find two disjoint sets $A(f)$ and $B(f)$ which partition the range of $f$ such that $C(f)=f^{-1}(A(f))$ and $D(f)=f^{-1}(B(f))$ both have cardinality $c$. Now find $\left\{E(f): f \in F_{0}\right\}$ a disjoint family of subsets of $c \times \mathbb{Z}$ such that $E(f)$ intersects both $C(f)$ and $D(f)$ in a set of cardinality $c$. Now choose $M(f) \subset c$ and a function $\rho_{f}: M(f) \rightarrow \mathbb{Z}$ such that, letting $N(f)=\left\{\left(\alpha, \rho_{f}(\alpha)\right): \alpha \in M(f)\right\}$, we have $N(f) \subset E(f)$ and $N(f)$ can be partitioned into two subsets of size $c$ with disjoint ranges contained in the partitions $R(f)$ and $S(f)$. Consider $\left\{\left(g^{-1}(R(f)) \cap N(f), g^{-1}(S(f)) \cap N(f)\right): g \in F\right\}$. This is a family of $c$ many partitions of $N(f)$. Choose $\pi: N(f) \leftrightarrow N(f)$ such that $\pi^{-1}$ of $\left(f^{-1}(R(f)) \cap N(f), f^{-1}(S(f)) \cap N(f)\right)$ does not equal any of the partitions. Now suppose $f \circ \pi=g$. Thus

$$
\begin{aligned}
& \left((f \circ \pi)^{-1}(R(f)) \cap N(f),(f \circ \pi)^{-1}(S(f)) \cap N(f)\right) \\
& \quad=\left(\pi^{-1}\left(f^{-1}(R(f)) \cap N(f)\right), \pi^{-1}\left(f^{-1}(S(f)) \cap N(f)\right)\right),
\end{aligned}
$$

which must equal $\left(g^{-1}(R(f)) \cap N(f), g^{-1}(S(f)) \cap N(f)\right)$.

This gives $\pi$ on each $N(f)$. We can extend $\pi$ to each $M(f) \times \mathbb{Z}$ by the rule $\pi(c, z)+i=\pi(c, z+i)$. Thus we have $\pi$ defined on $\bigcup\left\{M(f): f \in F_{0}\right\} \times \mathbb{Q}$ and can extend this arbitrarily to $c \times \mathbb{Z}$. The lemma is proved.

Proof of Theorem 6. Label the nodes of the Cantor tree by Lemma 5. Extend the branches $T$ of the Cantor tree to a maximal t.a.d. family $F$, and identify $T$ and $F-T$ by a two-to-one mapping to get another maximal t.a.d. family $G$.. Let $\mathscr{F}$ list the restrictions of the continuous functions on the $\psi$-space induced by $T(G)$ to the closed discrete set $T(G)$. Since $|\mathscr{F}|=c$, we can find $\pi: G \times \mathbb{Z} \rightarrow G \times \mathbb{Z}$ by Lemma 6 . Identify the copies of $T(G)$ in two copies of the induced $\psi$-space by $\pi$ to get the space $X$.

Suppose that $|\beta X-X|>1$. We deduce that $T(G)$ must have two disjoint infinite zero sets. These zero sets must be uncountable by Lemma 4 and thus of size $c$ by using the Cantor set. Thus there is a continuous function on the quotient $\psi$-space which is not almost constant. The traces $f$ and $f \circ \pi$ of this function onto the two $\psi$-spaces are both continuous. This is a contradiction.

However, $X$ does have an autohomeomorphism with no fixed points by Lemma 2. Lemma 3 completes the proof.

The referee has correctly observed that if we replace each isolated point of the space $X$ by a copy of the Cantor set (to be exact, take $X \times 2^{\omega}$ and collapse each $\{x\} \times 2^{\omega}$ to a singleton whenever $x$ is not isolated), then the resulting space $Y$ is a first countable homogeneous space with a reflection (translation) $f$ of $Y$ such that $\beta f$ has a fixed point. We just define $f$ to be the identity on the second coordinate and note that $Y$ is locally homeomorphic to the Cantor set.

The algebraic structure of any autohomeomorphism $f$ can be described by an $\omega$-sequence $\vec{f}$ of cardinals. When $n>0$, the $n$th entry is the number of orbits of length $n$ (thus the first entry is the number of fixed points). The 0th entry is the number of infinite orbits. 
Problem 2. For which sequences $\vec{\kappa}$ of cardinals is there an autohomeomorphism of some (regular) (compact Hausdorff) (metrizable) space $X$ such that $\vec{\kappa}=\vec{f}$ ?

Problem 2 may well have a specific instance which is quite difficult and fascinating.

Problem 3. For which sequences $\vec{\kappa}, \vec{\lambda}$ of cardinals is there an autohomeomorphism of some (first countable) completely regular space $X$ such that $\vec{\kappa}=\vec{f}$ and yet $\vec{\lambda}=\vec{\beta} f$ ?

A necessary condition is $(\forall n \in \omega) \kappa(n) \leq \lambda(n)$. The reader should notice that Problem 1 is just a specific instance (and quite possibly not the most interesting case) of Problem 3.

The problem can also be stated for specific spaces such as the rationals or irrationals.

Problem 4. For which sequences $\vec{\kappa}$ of cardinals is there an autohomeomorphism of the rationals (the irrationals) (the reals) such that $\vec{\kappa}=\vec{f}$ ?

\section{REFERENCES}

1. A. Blaszczyk and D. Y. Kim, A topological version of a combinatorial theorem of Katětov, Comment. Math. Univ. Carolin. 29 (1988), 657-663.

2. Eric K. van Douwen, $\beta X$ and fixed-point free maps, Topology Appl. 51 (1993), 191-195.

3. 1 (1976), 239-242.

4. Adam Krawczyk and Juris Steprāns, Continuous colourings of closed graphs, Topology Appl. 51 (1993), 13-26.

5. K. Mazur, Rationals and irrationals, unpublished note.

Department of Mathematics, York University, North York, Ontario, Canada M3J $1 \mathrm{P} 3$

E-mail address: watson $\bullet \mathrm{vm} 1$.yorku.ca 